http://dx.doi.org/10.35381/r.k.v5i2.1072

\title{
Diseño de una estación meteorológica automática para registrar las variables solar y eólica
}

\section{Design of an automatic weather station to record solar and wind variables}

\author{
Marcos Antonio Ponce-Jara \\ marcos.ponce@uleam.edu.ec \\ Universidad Laica Eloy Alfaro de Manabí, Manta \\ Ecuador \\ https://orcid.org/0000-0002-4450-4740 \\ Giselle Velásquez-Figueroa \\ giselle.velasquez@uleam.edu.ec \\ Universidad Laica Eloy Alfaro de Manabí \\ Ecuador \\ https://orcid.org/0000-0002-4494-6784 \\ David Tonato-Peralta \\ ctonato@inamhi.gob.ec \\ Universidad Laica Eloy Alfaro de Manabí, Manta \\ Ecuador \\ https://orcid.org/0000-0003-4188-5354 \\ George Paredes-Morillo \\ georgejoseth1615@gmail.com \\ Universidad Laica Eloy Alfaro de Manabí, Manta \\ Ecuador \\ https://orcid.org/0000-0001-5298-0117
}

Recepción: 10 de julio 2020

Revisado: 29 de agosto 2020

Aprobación: 20 de septiembre 2020

Publicación: 01 de octubre 2020 


\title{
RESUMEN
}

La investigación tuvo por objetivo diseñar una estación meteorológica automática para registrar las variables solar y eólica. Se desarrolló desde la metodología de tipo descriptiva documental con diseño no experimental, lo cual, permitió en una primera fase, analizar datos meteorológicos con la finalidad de diagnosticar la necesidad de contar con el diseño de una propuesta de una estación meteorológica. Se observa que la radiación en este mes se osciló entre los 5.000 y $7.000 \mathrm{Wh} /\left(\mathrm{m}^{2}\right.$-día), con algunas excepciones, llegando incluso a sobrepasar los $8.000 \mathrm{Wh} /\left(\mathrm{m}^{2}\right.$-día). Es de notar que hubo días con una bajísima radiación solar dadas las lluvias y fuerte nubosidad encontradas en este mes. La estación meteorológica automática diseñada, evidencia una gran ventaja frente a las estaciones meteorológicas convencionales; esta puede ser usada en zonas con poca accesibilidad o en ausencia de red eléctrica.

Descriptores: Condiciones metereológicas; control meteorológico; bioclimatología. (Palabras tomadas del Tesauro UNESCO).

\begin{abstract}
The objective of the research was to design an automatic meteorological station to record the solar and wind variables. It was developed from a descriptive documentary methodology with a non-experimental design, which, in a first phase, allowed analyzing meteorological data in order to diagnose the need to have the design of a proposal for a meteorological station. It is observed that the radiation in this month ranged between 5,000 and 7,000 Wh / (m2-day), with some exceptions, even exceeding 8,000 Wh / (m2day). It is noteworthy that there were days with very low solar radiation due to the rains and heavy cloud cover found this month. The automatic weather station designed, shows a great advantage over conventional weather stations; This can be used in areas with little accessibility or in the absence of a power grid.
\end{abstract}

Descriptors: Weather; weather modification; bioclimatology. (Words taken from the UNESCO Thesaurus). 


\section{INTRODUCCIÓN}

El planeta tierra a lo largo de sus millones de años de existencia ha experimentado cambios climatológicos importantes; por ejemplo, la última glaciación de la que se tiene registro de hace aproximadamente 10000 años. (Ureña-Elizondo, 2017). La meteorología es la ciencia interdisciplinaria de la física de la atmosfera, que estudia el estado del tiempo, el medio atmosférico, los fenómenos producidos y las leyes que lo rigen (Barriga, et al., 2015). En este proyecto se plantea contribuir al estudio de la meteorología con la construcción de una estación meteorológica, a fin de poder tomar medidas y realizar el seguimiento de las siguientes variables atmosféricas: radiación solar, velocidad del viento, dirección del viento.

El proceso de mediciones meteorológicas está directamente relacionado con la implementación de una estación meteorológica, se busca tener acceso a de los sensores instalados desde cualquier parte del mundo a través de una página web (Estévez, 2011). La medición de las variables meteorológicas se realiza mediante la observación de cualquier zona geográfica, es en esencia una medición física, que se efectúa de manera manual en las horas sinópticas, esta información es registrada diariamente en una ficha meteorológica. Mediante la ingeniería y tecnología electrónica, el observador humano puede ser en algunos casos sustituido parcial o completamente por una máquina, optimizando el proceso de medición de los distintos parámetros meteorológicos (RuizAyala, et al., 2018). En este sentido la configuración propia del sistema es elemental, la formación de este solo depende del modelo que se especifique con los requerimientos de la implementación donde sea aplicado.

En complemento a lo descrito, se comenta la experiencia del Instituto Nacional de Meteorología e Hidrología (INAMHI), con la necesidad de disponer de información en tiempo real para los sistemas de vigilancia meteorológica y para los sistemas de alerta temprana, en el 2003 se iniciaron los primeros programas para la creación de una red de meteorología automática. Ya en el 2013 se contaba con 91 estaciones meteorológicas automáticas, las cuales contaban con comunicación satelital y General Packet Radio 
Service (GPRS) en tiempo real para enlazarse con los servidores del INAMHI (INAMHI, 2013).

Para el 2019 se destaca que en Manabí solo se encuentran 5 estaciones meteorológicas habilitadas, como se muestra en la Figura 1, corresponden a las estaciones M0168, M1217, M0162, M1208, M1233.

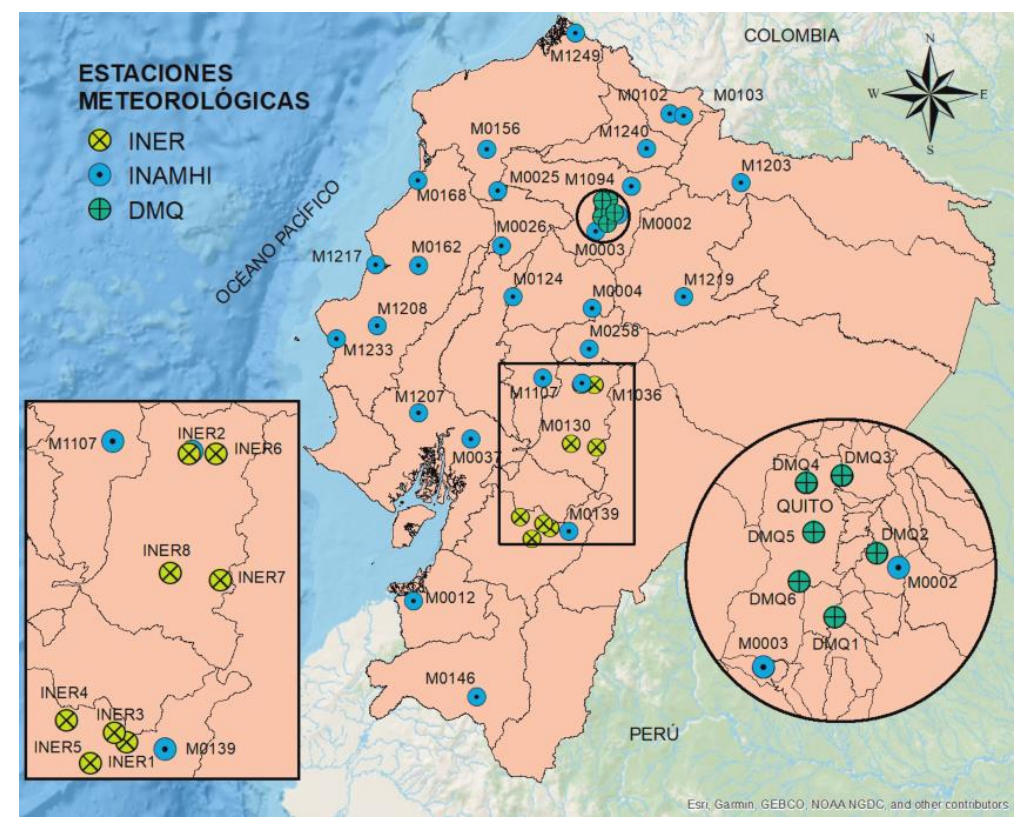

Figura 1. Red de estaciones meteorológicas Ecuador 2019.

Fuente: Vaca-Revelo \& Ordóñez (2019).

\section{Potencial Solar y Eólico en Manabí}

Según los datos del Altas Solar y Eólico (CONELEC, 2008), la provincia de Manabí posee un elevado potencial solar, y existen algunas zonas de gran interés donde la energía eólica puede ser aprovechada mediante tecnología mini eólica y plantas de mayor extensión. 


\section{Potencial Solar}

La provincia de Manabí cuenta con uno de los niveles más elevados de radiación solar incidente en el país, con valores que pueden alcanzar alrededor de $4700 \mathrm{Wh} / \mathrm{m} 2 /$ día (CONELEC, 2008). Sin embargo, el comportamiento de esta variable no es uniforme y posee variaciones en todo el espacio geográfico de la provincia que vale la pena estudiar para tener un perfil más exacto de su comportamiento. Al mismo tiempo, pese a ser un recurso abundante, su uso en la provincia se limita a una instalación solar fotovoltaica de $1 \mathrm{MW}$ en el cantón Jaramijó y $0.5 \mathrm{MW}$ en Atacames. La Figura 2 muestra el potencial solar diario promedio anual en la provincia de Manabí.

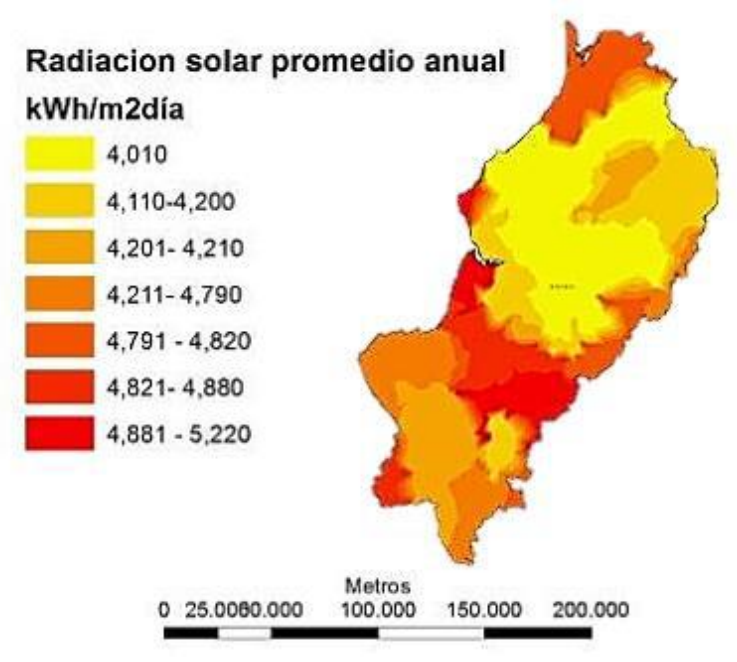

Figura 2. Mapa de radiación solar promedio anual en Manabí.

Fuente: Saltos Arauz, et al., (2017). 


\section{Potencial Eólico}

El mayor potencial eólico en Ecuador se encuentra ubicado en la región andina, en las provincias de Carchi, Imbabura, Chimborazo, Cañar y Loja, donde se estima un potencial de aproximadamente 1670 MW. En menor proporción se han ubicado regiones costeras como la península de Santa Elena, el Aromo o partes de las costas de Esmeraldas (MEER, 2013). En la Figura 3 se observa el mapa de velocidad del viento de la provincia de Manabí del año 2016, así como las ciudades y pueblos ubicados en el litoral, donde mayor intensidad de viento se registra según estudios sobre datos estadísticos con imágenes satelitales.

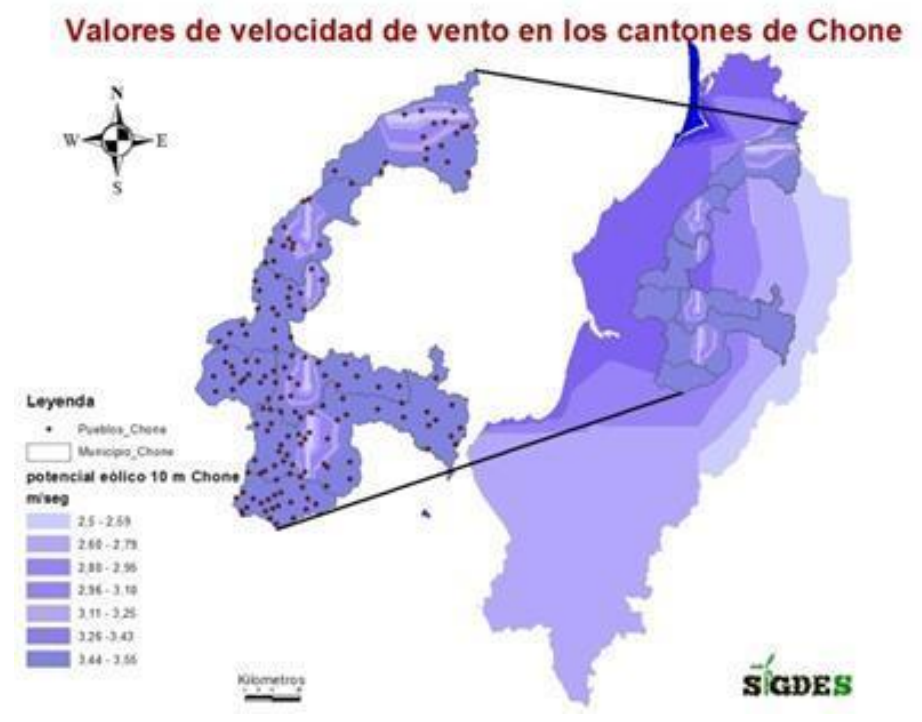

Figura 3. Mapa de velocidad promedio Anual en Manabí.

Fuente: Rodríguez-Gámez, et al. (2017).

Además, según la Corporación para Investigación Energética (CIE, 2017), en Manabí existen dos zonas de importancia para la generación eléctrica mediante tecnología eólica a gran escala, las cuales están situadas en el Aromo (cantón Manta) y cerro de Montecristi (cantón Montecristi). Se determinó que las velocidades promedias a $80 \mathrm{~m}$ de altura en estos puntos podrían alcanzar los $6.63 \mathrm{~m} / \mathrm{s}$ y $5.88 \mathrm{~m} / \mathrm{s}$ respectivamente.

En razón de lo descrito, la investigación tuvo por objetivo diseñar una estación 
meteorológica automática para registrar las variables solar y eólica.

\section{MÉTODO}

La investigación se desarrolló desde la metodología de tipo descriptiva documental con diseño no experimental, lo cual, permitió en una primera fase, analizar datos meteorológicos con la finalidad de diagnosticar la necesidad de contar con el diseño de una propuesta de una estación meteorológica en una segunda fase de trabajo. De ese modo, se procedió a construir la estructura tanto en hardware y software, para lograr la adquisición de datos solares y eólicos, los que se acondicionaron mediante el dispositivo Raspberry $\mathrm{Pi}$, en función de datalogger, este tipo de estudio localmente no se ha realizado con la profundidad ni el aval de que tiene el desarrollo de esta investigación.

\section{ANÁLISIS DE LOS RESULTADOS}

A continuación, se analizan los datos recolectados de radiación solar, velocidad y dirección de viento para los meses de febrero a abril.

En el Gráfico 1, se muestran datos de radiación solar expresados en Wh/(m²-día) del mes de febrero recolectados por la estación meteorológica. Se observa que la radiación en este mes se osciló entre los 5.000 y $7.000 \mathrm{Wh} /\left(\mathrm{m}^{2}\right.$-día), con algunas excepciones, llegando incluso a sobrepasar los $8.000 \mathrm{Wh} /\left(\mathrm{m}^{2}\right.$-día). Es de notar que hubo días con una bajísima radiación solar dadas las lluvias y fuerte nubosidad encontradas en este mes. 
Revista Arbitrada Interdisciplinaria KOINONIA

Año V. Vol V. №10. Julio - Diciembre 2020

Hecho el depósito de Ley: FA2016000010

ISSN: 2542-3088

FUNDACIÓN KOINONIA (F.K). Santa Ana de Coro. Venezuela.

Marcos Antonio Ponce-Jara; Carlos Velásquez-Figueroa; David Tonato-Peralta;

George Paredes-Morillo

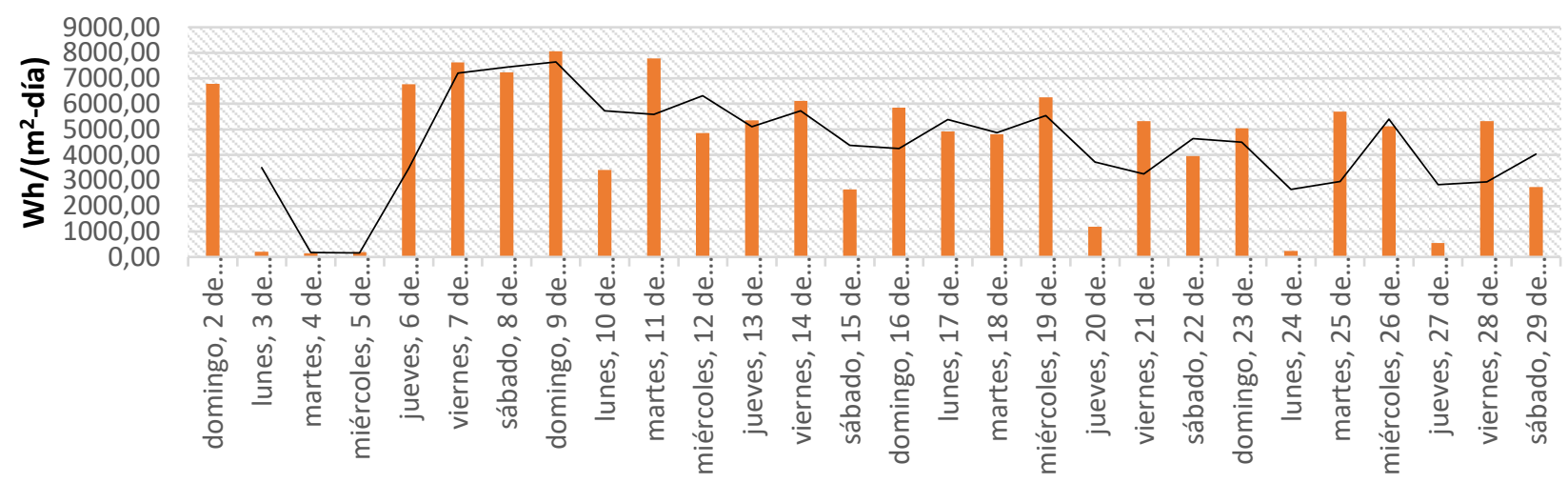

FECHA POR DÍA DEL MES

Gráfico 1. Datos de radiación Solar del mes de febrero 2020.

Fuente: https://meteorologia.uleam.edu.ec/

En el Gráfico 2 se muestran datos de radiación solar del mes de marzo. Durante este mes obtuvieron mejores resultados de radiación solar con respecto al mes anterior. Los datos obtenidos muestran un mes más estable y con menos oscilaciones propios del mes de vacaciones de la costa ecuatoriana. La mayor parte de los días oscilaron entre los 5.000 y un poco más de $8.000 \mathrm{Wh} /\left(\mathrm{m}^{2}\right.$-día), siendo esta una cantidad muy buena de energía que se pudo aprovechar.

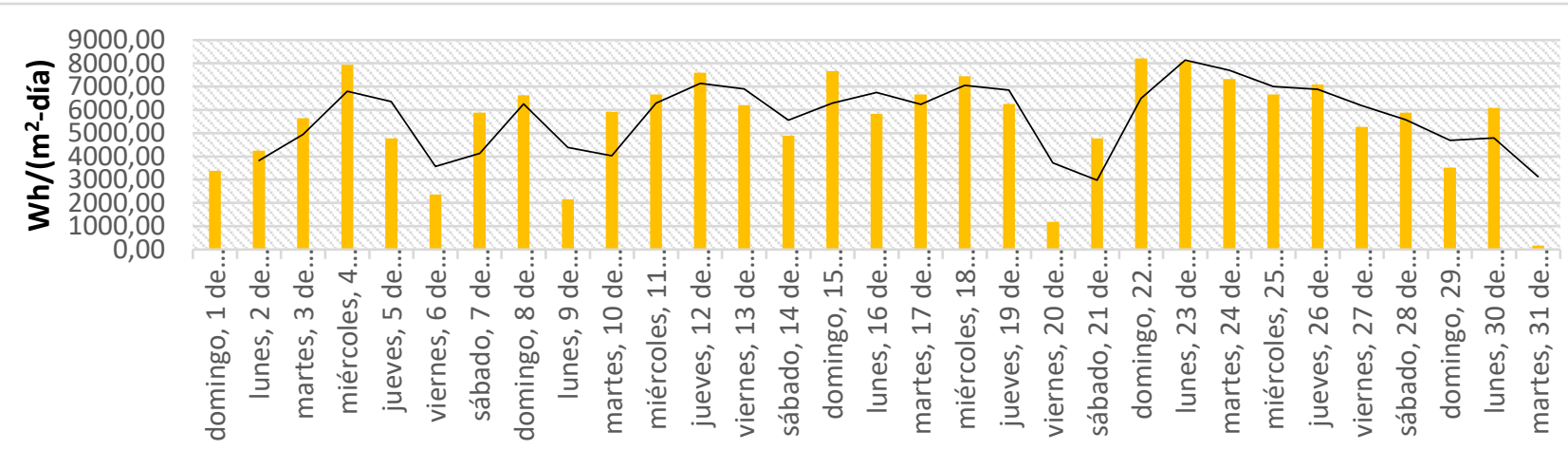

FECHA POR DÍA DEL MES

Gráfico 2. Datos de Radiación Solar del mes de marzo 2020.

Fuente: https://meteorologia.uleam.edu.ec/ 
Revista Arbitrada Interdisciplinaria KOINONIA

Año V. Vol V. N 10 . Julio - Diciembre 2020

Hecho el depósito de Ley: FA2016000010

ISSN: 2542-3088

FUNDACIÓN KOINONIA (F.K). Santa Ana de Coro. Venezuela.

Marcos Antonio Ponce-Jara; Carlos Velásquez-Figueroa; David Tonato-Peralta;

George Paredes-Morillo

En el Gráfico 3 se muestran datos de radiación solar del mes de abril. De manera general este mes osciló entre los 5.000 y $7.500 \mathrm{Wh} /\left(\mathrm{m}^{2}\right.$-día) pero se observa que hubo picos mayores a los $9.000 \mathrm{Wh} /(\mathrm{m} 2$-día) siendo este mes el más alto en cuanto a radiación solar de los presentados.

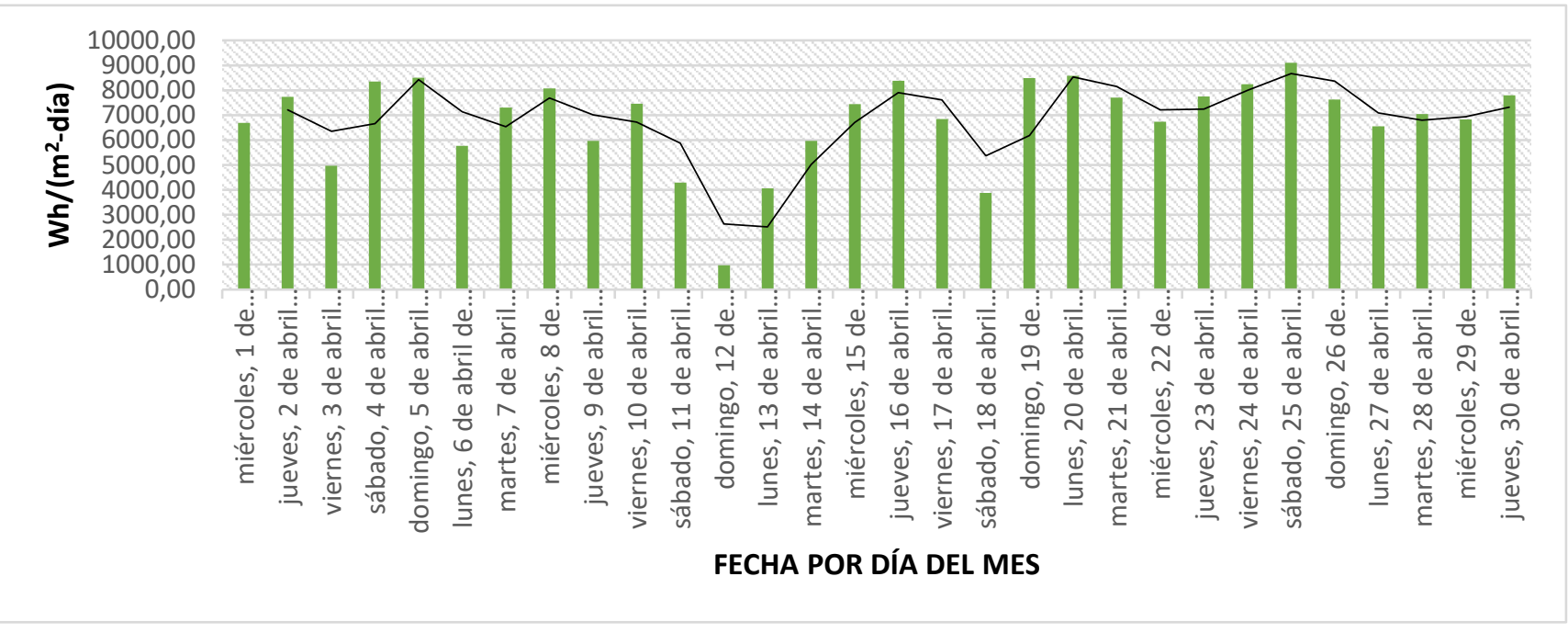

Gráfico 3. Datos de Radiación Solar del mes de abril 2020.

Fuente: https://meteorologia.uleam.edu.ec/

En el Gráfico 4 se muestra la media de radiación solar medida durante los meses mencionados; estos sugieren la viabilidad de usar tecnología solar fotovoltaica dada la

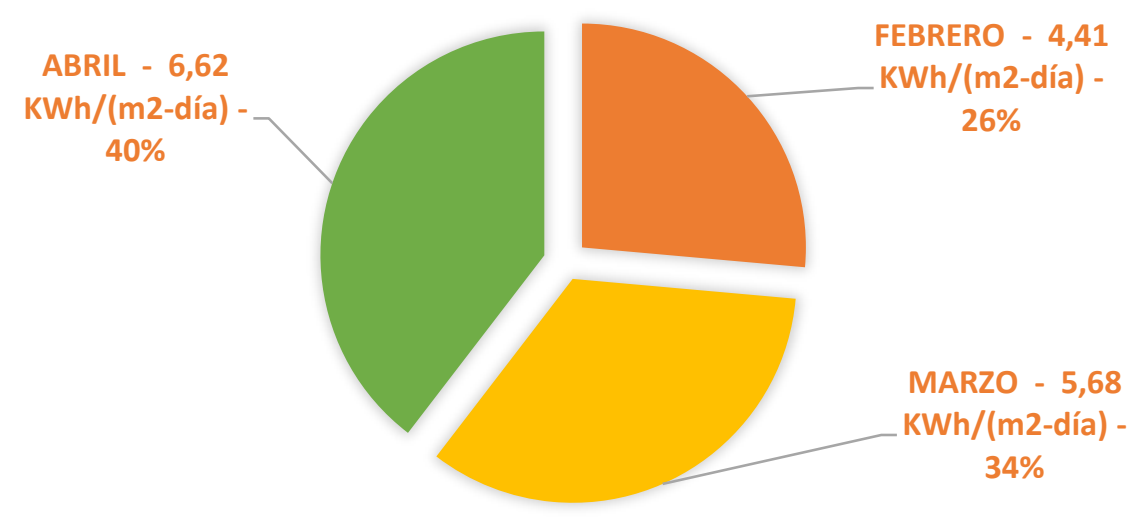


alta radiación solar incidente sobre este territorio; el siguiente paso corresponde a realizar un estudio técnico-económico que respalde esta asunción. Aunque en el mes de abril se presentaron picos muy elevados de Radicación Solar el mes que generó efectivamente más radiación solar fue el mes de marzo.

Gráfico 4. Datos de Porcentajes de Radiación Solar 2020.

Fuente: https://meteorologia.uleam.edu.ec/

En cuanto a los datos de dirección del viento, en el Gráfico 5 se observa un gráfico radial con el objetivo de identificar de mejor manera la frecuencia de dirección del viento entre los meses de febrero - abril. Como se observa, la direccion de viento es predominate hacia el sur, donde se encuentran la mayor cantidad de datos (entre los $276^{\circ}$ y $289^{\circ}$ ), además, existe una cantidad de datos considerable entre $159^{\circ}$ y $237^{\circ}$. 
Revista Arbitrada Interdisciplinaria KOINONIA

Año V. Vol V. N 10 . Julio - Diciembre 2020

Hecho el depósito de Ley: FA2016000010

ISSN: 2542-3088

FUNDACIÓN KOINONIA (F.K). Santa Ana de Coro. Venezuela.

Marcos Antonio Ponce-Jara; Carlos Velásquez-Figueroa; David Tonato-Peralta;

George Paredes-Morillo

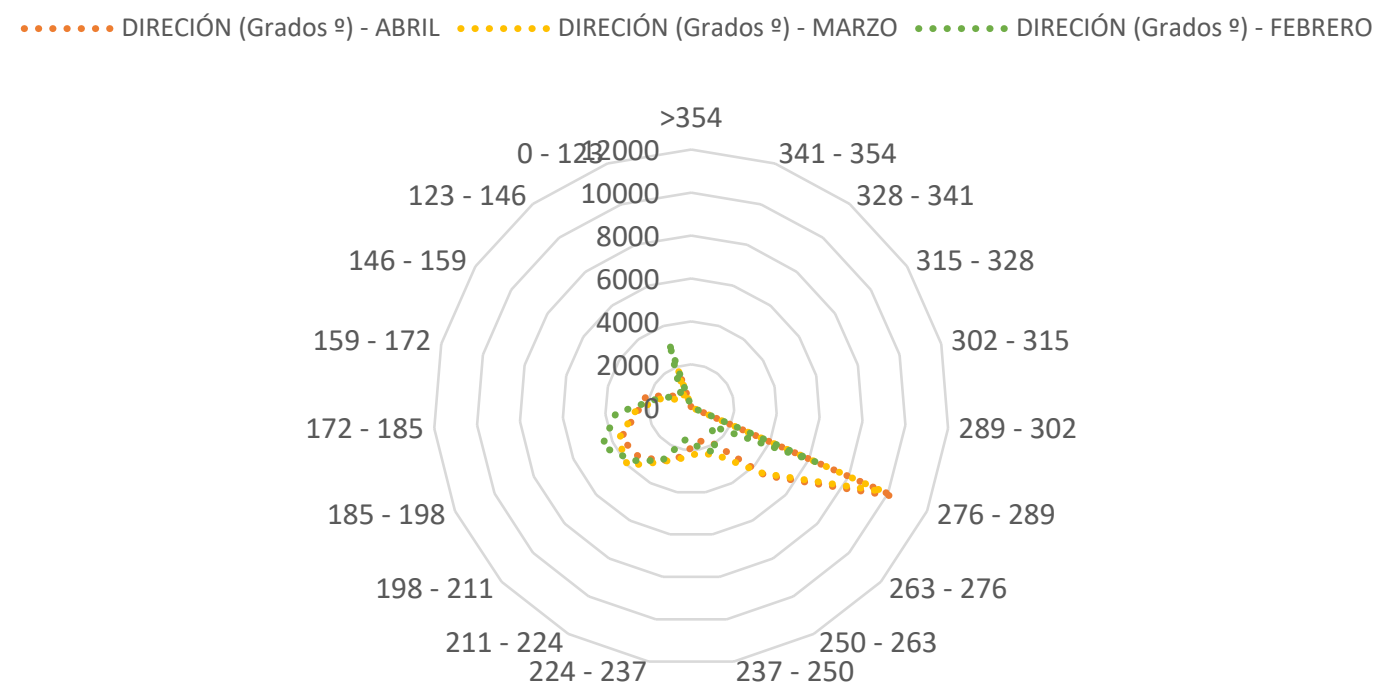

Gráfico 5. Datos de Dirección del viento febrero-abril 2020.

Fuente: https://meteorologia.uleam.edu.ec/

Por otro lado, en cuanto a la velocidad del viento, en el Gráfico 6 se muestran los datos de los meses febrero-abril tabulados por cantidad de datos y velocidad de viento. Se observa una correlación alta entre mayor cantidad de datos y bajas velocidades, y menor cantidad de datos para velocidades superiores a $3 \mathrm{~m} / \mathrm{s}$. 
Revista Arbitrada Interdisciplinaria KOINONIA

Año V. Vol V. №10. Julio - Diciembre 2020

Hecho el depósito de Ley: FA2016000010

ISSN: 2542-3088

FUNDACIÓN KOINONIA (F.K). Santa Ana de Coro. Venezuela.

Marcos Antonio Ponce-Jara; Carlos Velásquez-Figueroa; David Tonato-Peralta;

George Paredes-Morillo

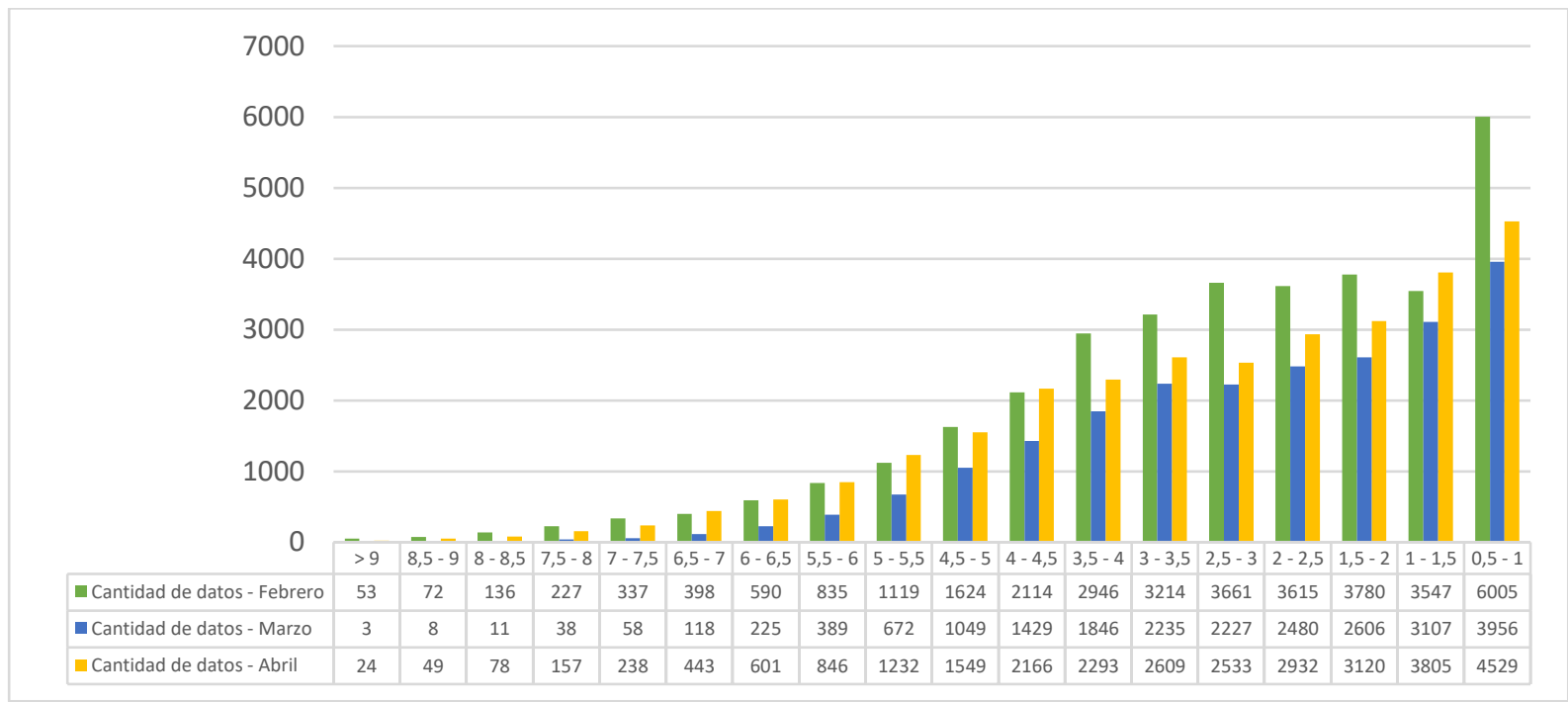

Gráfico 6. Comparativa Datos de Velocidad del viento de los meses de febrero, marzo y abril 2020.

Fuente: https://meteorologia.uleam.edu.ec/

Por lo general, para poder aprovechar la energía del viento a través de tecnología mini eólica es necesario contar con velocidades superiores a $3 \mathrm{~m} / \mathrm{s}$, por tanto, estos datos sugieren que existe una buena cantidad de energía del viento que puede ser aprovechada para producir energía eléctrica mediante tecnología mini eólica. 


\section{PROPUESTA DE LA ESTACIÓN METEOROLÓGICA AUTOMÁTICA (EMA)}

Dentro de estas tipologías, una estación meteorológica automática (EMA) se define como "una estación meteorológica en la que las observaciones se efectúan y transmiten automáticamente" (OMM, 2017). Por lo que comprende una instalación en la que se tiene una serie de instrumentos destinados a la recolección y registro de las variables meteorológicas según su tipo, sean climáticas, sinópticas o marinas.

Las mediciones realizadas con una EMA son leídas y registradas por una unidad central de datos o "datalogger", las cuales pueden ser procesadas por el propio dispositivo o externamente. El principal objetivo de estas estaciones es aumentar el número y la fiabilidad de las observaciones en superficie, y lo consiguen (OMM, 2017).

Para este cometido, mediante un convenio de colaboración con el INAMHI, se procedió a calibrar y validar tanto los sensores como datalogger. El diseño propuesto es el que se muestra en la figura 4; este está compuesto de 3 sensores: Piranómetro, Anemómetro y Veleta. Las señales provenientes de estos sensores pasan por un módulo conversor analógico digital $(A D C)$ para acondicionar las señales antes de entrar en la unidad central de procesamiento o datalogger que para este diseño se escogió una RaspBerry Pi. Cada sensor es leído al mismo tiempo por ende el proceso de lectura dura un minuto, donde se ejecutan filtros digitales en donde se establece un promedio de los valores para estos ser enviados tanto a los servidores del INAMHI como a los de la ULEAM para ser almacenados.

Conjuntamente, son mostrados en tiempo real en un aplicativo web de libre acceso a la comunidad universitaria donde son más fáciles de visualizar los cambios con dirección (ULEAM, 2020). Complementariamente lleva incorporado un sistema solar fotovoltaico para alimentar a la estación meteorológica en ausencia de red eléctrica. En la figura 4 se indica el flujo de trabajo de la estación meteorológica.

\section{$+$}


Revista Arbitrada Interdisciplinaria KOINONIA

Año V. Vol V. №10. Julio - Diciembre 2020

Hecho el depósito de Ley: FA2016000010

ISSN: 2542-3088

FUNDACIÓN KOINONIA (F.K). Santa Ana de Coro. Venezuela.

Marcos Antonio Ponce-Jara; Carlos Velásquez-Figueroa; David Tonato-Peralta;

George Paredes-Morillo

En la figura 4 se observa el diseño físico de la estructura y localización de los sensores.

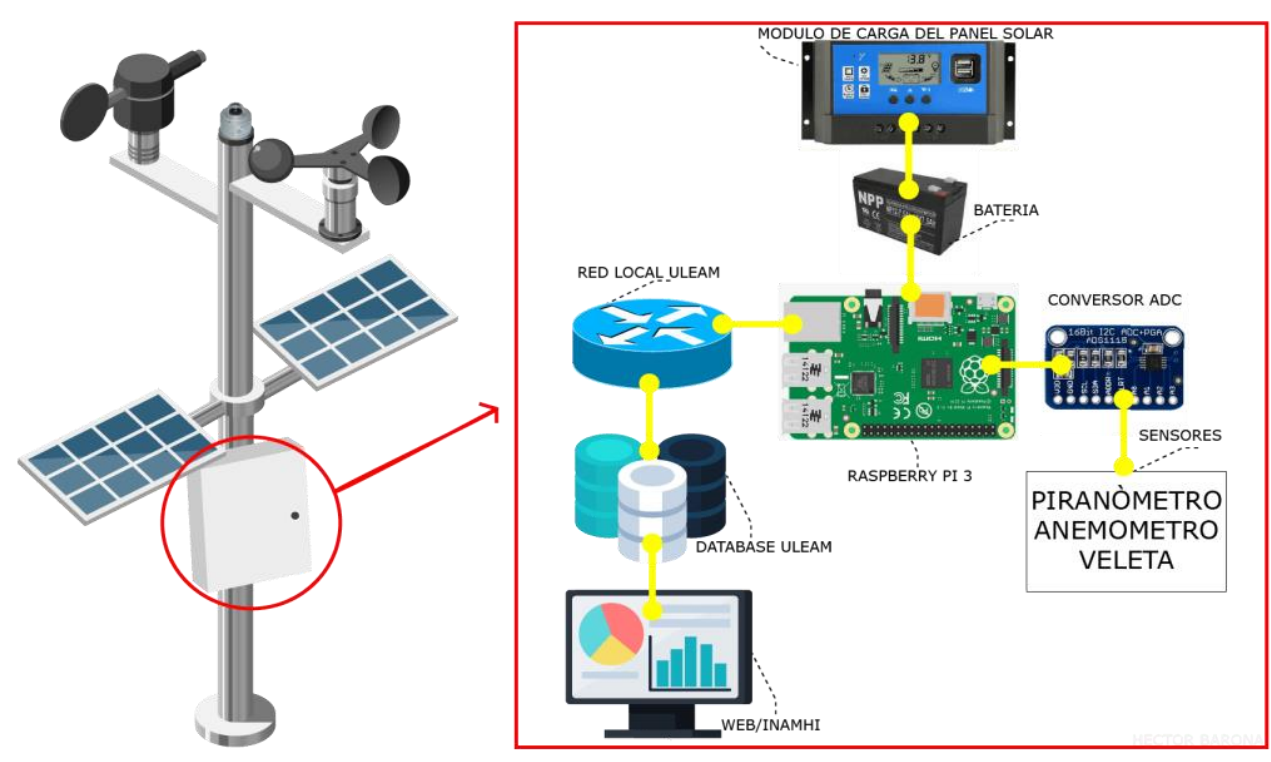

Figura 4. Esquema global de la estación meteorológica automática.

Fuente: Elaboración propia. 


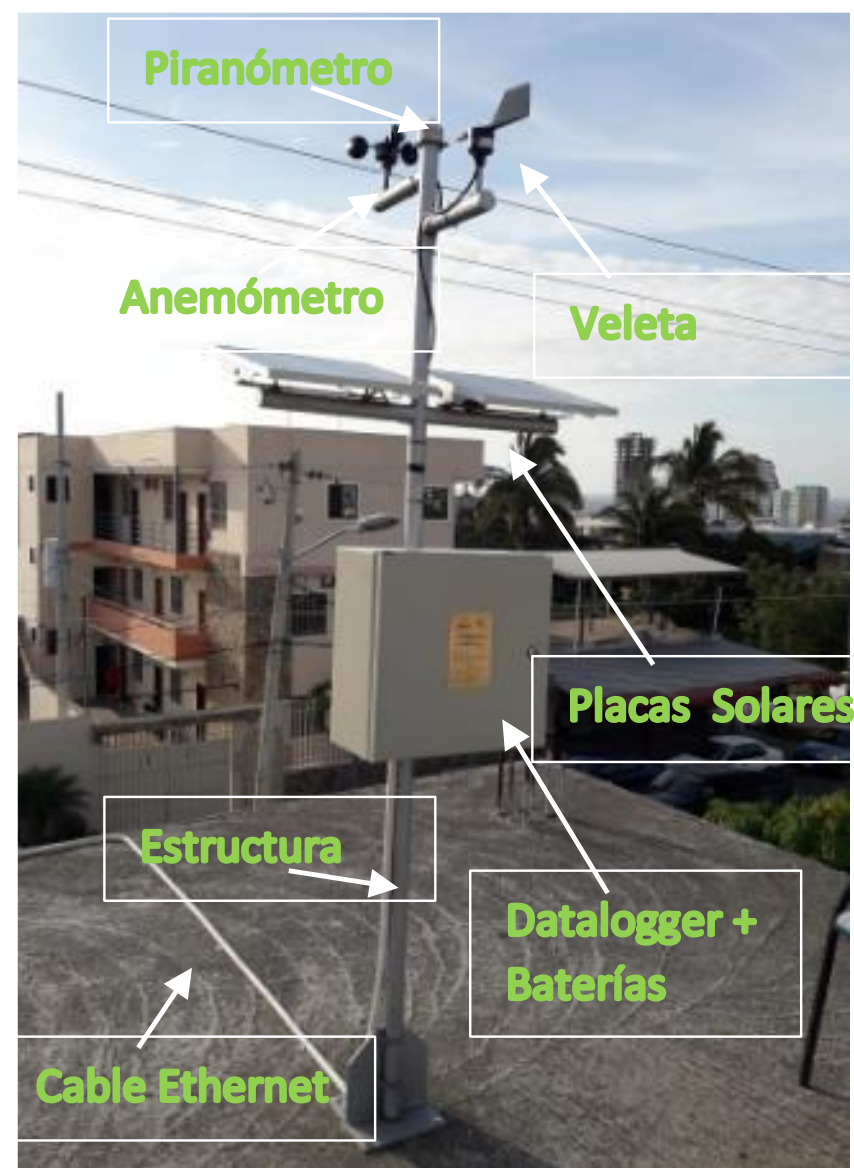

Figura 5. Prototipo - Estructura Física de la estación meteoróloga automática. Fuente: Elaboración propia. 
El datalogger es uno de los dispositivos más importantes de la instalación y representa la unidad central de procesamiento para la adquisición de datos de los sensores y su conversión en formato legible por la computadora; se encarga además del almacenamiento y envió de la información remotamente mediante la utilización de algoritmos específicos para tal cometido. Por lo general, los precios de los dispositivos encargados de estas actividades son muy elevados, en este artículo se propone como alternativa la utilización de un microcomputador RaspBerry Pi 3B como datalogger, en la figura 6 se indican las características del dispositivo utilizado en este proyecto.

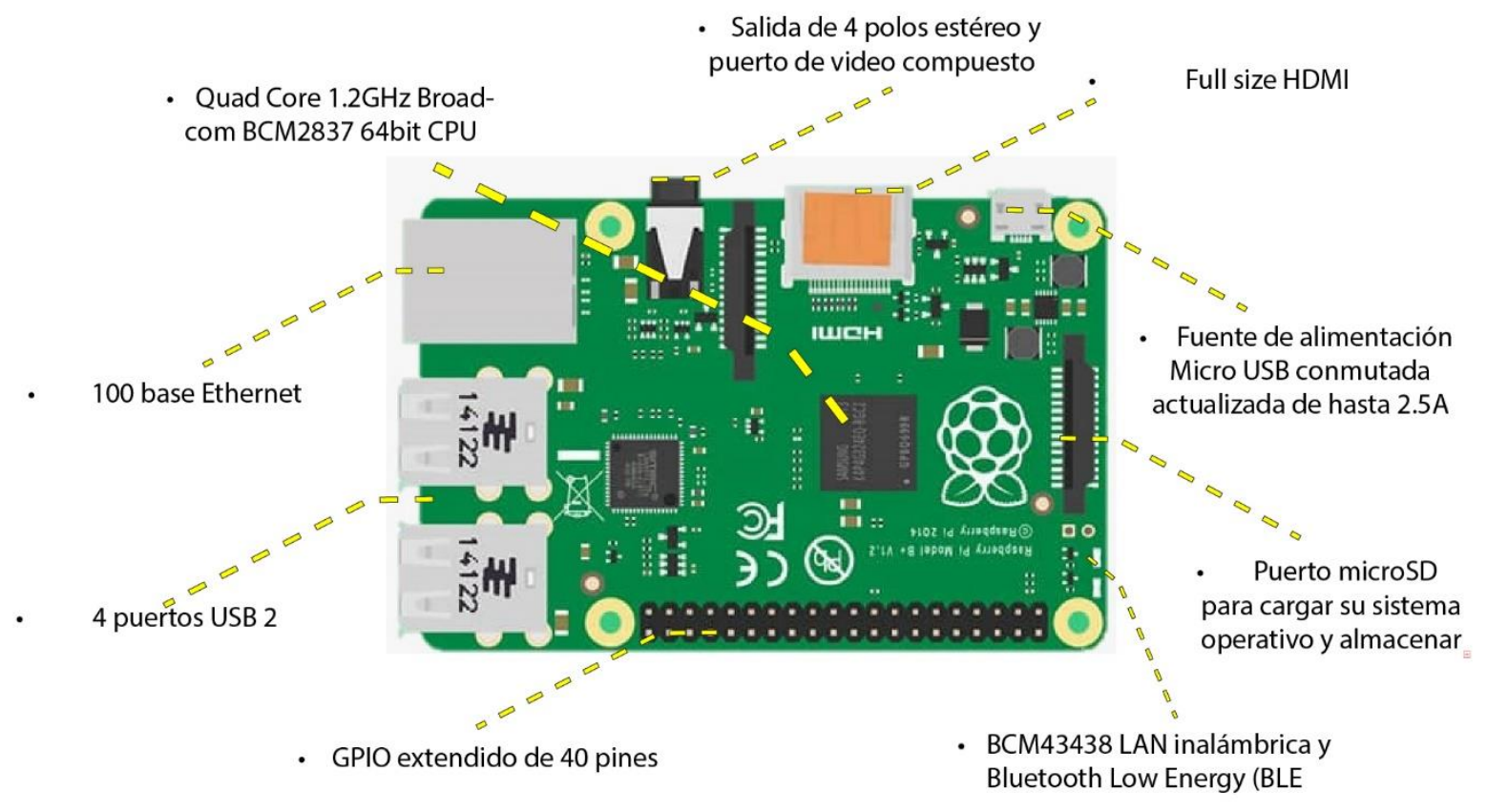

Figura 6. Características de la RaspBerry Pi 3 Modelo B.

Fuente: Elaboración propia a partir de datos de Pi, R. (2015)

\section{Sensores para la medición de las variables meteorológicas}

Una estación meteorológica debe ser capaz de medir variables analógicas y digital, para 
este diseño se dotó de sensores (analógicos y digitales) para medir radiación solar, dirección y velocidad de viento. La radiación solar y la dirección del viento es medida por sensores analógicos, los cuales utilizan un conversor analógico digital (A/D) para adecuar la señal antes de entrar en la RaspBerry Pi 3B; el conversor se comunica con la RaspBerry a través del protocolo de comunicación I2C. Por otro lado, el anemómetro es un sensor que proporciona una señal digital que se conecta directamente al puerto GPIO de la RaspBerry Pi 3B.

\section{Anemómetro y veleta}

El sensor encargado de medir la velocidad del viento es el anemómetro NRG \#40GH se conecta directamente a una de las entradas digitales del puerto GPIO de la RaspBerry Pi3B; este sensor proporciona una señal digital en forma de tren de pulsos, generando un pulso cada vez que se cierra el circuito por cada vuelta generada. Internamente tiene un imán tetrapolar induce un voltaje de la onda sinusoidal en una bobina, produciendo una señal de salida con la frecuencia proporcional a la velocidad del viento. la velocidad que se registre es proporcional al número de vueltas que se produzca en un segundo, según su hoja de características, (NRG, 2015).

La dirección del viento es registrada mediante el sensor NRG \#200P, el cual es un sensor analógico alimentado a 5 voltios $(5 \mathrm{~V})$ que provee una tensión proporcional a los grados en los que se encuentre la veleta. Para poder acondicionar la señal antes de ser leída por la RaspBerry Pi3B es necesaria la utilización de un conversor A/D. La forma que se determina la posición viene definitiva por la siguiente fórmula: Dirección [grados de rotación] $=$ Vout ${ }^{\star} 360^{\circ} / 5$ (voltios).

\section{Piranómetro}

El piranómetro es el encargado de medir la radiación solar incidente global que llega a la 
superficie de la tierra; se trata de un sensor basado en una termopila que genera una señal en milivoltios $(\mathrm{mV})(0-50 \mathrm{mV})$, que puede medirse directamente en un datalogger sin necesidad de una fuente externa de alimentación. Para este sensor es necesario el uso del conversor A/D de mínimo 16 bits de velocidad, para acondicionar la señal antes de entrar en la RaspBerry Pi3B. Una vez recibida la señal eléctrica correspondiente a la radiación solar, la fórmula que se utiliza para determinar la radiación solar es: Radiación Solar $[\mathrm{W} / \mathrm{m} 2]=$ Vout ${ }^{\star} 2000\left(\mathrm{w} / \mathrm{m}^{2}\right) / 0.05$ (voltios)

\section{CONCLUSIONES}

La estación meteorológica automática diseñada, evidencia una gran ventaja frente a las estaciones meteorológicas convencionales; esta puede ser usada en zonas con poca accesibilidad o en ausencia de red eléctrica, por lo cual es vital el diseño de un sistema solar fotovoltaico que asegure el abasto energético por un largo periodo.

En su funcionamiento el datalogger es el elemento más importante de una EMA ya que es el encargado de recoger y procesar las señales medidas por los diferentes sensores meteorológicos. Para poder utilizar este sistema, es necesario la calibración de los instrumentos de medida; por ende, se diseñó un proceso de lectura en paralelo, es decir que cada sensor se lee por igual y a tiempo real, para obtener resultados fiables.

La aparición de microcomputadores de bajo costo como la RaspBerry Pi3B, ofrece una buena alternativa para el diseño de un datalogger para usarlo en el campo de la meteorología. El mismo que está compuesto por múltiples puertos de comunicación (USB, Serial, SPI, I2C, HMI, Ethernet) los cuales permiten interactuar con el dispositivo de forma amigable y remota; permitiendo que esta estación sea totalmente funcional, admite la ampliación de más sensores y permite leerlos en tiempo real, sin riesgo de perder datos.

De igual forma, el uso de servidores web para almacenar la información medida es de suma importancia para obtener un respaldo seguro a largo plazo. El tiempo de prueba determino el buen funcionamiento de este prototipo, no obstante, se pueden mejorar. 
La información de obtenida de radiación, dirección velocidad del viento aún no es concluyente, pero da una idea de la viabilidad de aprovechar este recurso para generar proyectos energéticos e incentivar el uso de energías renovables no convencionales.

Sin embargo, se requiere de un estudio de factibilidad técnico-económico, que utilice las variables caracterizadas en este proyecto, para justificar la implementación de este tipo de tecnologías.

\section{FINANCIAMIENTO}

No monetario.

\section{AGRADECIMIENTO}

A la Universidad Laica Eloy Alfaro de Manabí y al Instituto Nacional de Meteorología e Hidrología, Ecuador; por apoyar la investigación.

\section{REFERENCIAS CONSULTADAS}

Barriga, M, Viscaíno, J, \& Recalde, C. (2015). Implementación De Una Red De Estaciones Meteorológicas Utilizando Transmisión GPRS En La Región Centro Andina Ecuatorial [Implementation of a network of meteorological stations using GPRS transmission in the central equatorial andean region]. $X$ Congreso de Ciencia $y$ Tecnología ESPE 2015, ISSN: 1390-4671, 10(1); 209-214.

CIE. (2017). Corporación para la Investigación Energética. Estudio de prefactibilidad eólica en la Provincia de Manabí. [Wind pre-feasibility study in the Province of Manabí]. Recuperado https://n9.cl/ith7

CONELEC. (2008). Consejo Nacional De Electricidad. Atlas solar del Ecuador. [National Electricity Council. Solar Atlas of Ecuador]. https://n9.cl/s47n

Estévez, J, Gavilán, P, \& Giráldez, J.V. (2011). Guidelines on validation procedures for meteorological data from automatic weather stations. Journal of Hydrology, 402, 144-154. https://doi.org/10.1016/j.jhydrol.2011.02.031 
INAMHI. (2013). INAMHI 52 años haciendo historia (1961-2013). [NIMH 52 years making history (1961-2013)]. https://n9.cl/ issuu

NRG S. (2015). NRG 40C Anemometer. [online] Recuperado de https://n9.cl/p9ars

Organización Meterológica Mundial, OMM. (2017). Guía del Sistema Mundial de Observación [Guide to the Global Observing System]. Recuperado de https://n9.cl/m0j5

Pi, R. (2015). Modelo Raspberry pi 3 b. [online]. Recuperado de https://n9.cl/3fl4

Rodríguez-Gámez, M., Vázquez-Pérez, A., \& Saltos-Arauz, M. (2017). Eje 06-06 la ordenación territorial y las fuentes renovables de energía [Axis 06-06 land use planning and renewable energy sources]. Memorias Y Boletines De La Universidad Del Azuay, 1(XVI), 287 - 292.

Ruiz-Ayala, D., Vides-Herrera, C., \& Pardo-García, A. (2018). Monitoreo de variables meteorológicas a través de un sistema inalámbrico de adquisición de datos [Monitoring of meteorological variables through a wireless data acquisition system]. REVISTA DE INVESTIGACIÓN, DESARROLLO E INNOVACIÓN, 8(2), 333-341. https://doi.org/10.19053/20278306.v8.n2.2018.7971

Universidad Laica Eloy Alfaro De Manabi, ULEAM (2020). Estación meteorológica automática [Automatic weather station]. Recuperado de https://meteorologia.uleam.edu.ec/

Ureña-Elizondo, F. (2017). Utilización de estaciones meteorológicas automáticas como nueva alternativa para el registro y transmisión de datos [Use of automatic meteorological stations as a new alternative for data recording and transmission. Postgraduate and Society Magazine]. Revista Posgrado y Sociedad, 11(1). 33-49. https://doi.org/10.22458/rpys.v11i1.1881

Vaca-Revelo, D. and Ordóñez, F., (2019). Mapa Solar Del Ecuador 2019 [Solar Map Of Ecuador 2019].1st ed. Quito, Ecuador: Grupo de Investigación SCINERGY. Recuperado de https://n9.cl/pzpop 
Revista Arbitrada Interdisciplinaria KOINONIA

Año V. Vol V. N 10 . Julio - Diciembre 2020

Hecho el depósito de Ley: FA2016000010

ISSN: 2542-3088

FUNDACIÓN KOINONIA (F.K). Santa Ana de Coro. Venezuela.

Marcos Antonio Ponce-Jara; Carlos Velásquez-Figueroa; David Tonato-Peralta;

George Paredes-Morillo

Vázquez-Pérez, A., Saltos-Arauz, W., Rodríguez-Gámez, M., Castro-Fernández, M., \& Nieto-Castro, V. (2017). Sistemas de información geográfica y microrredes [Geographic information systems and microgrids]. Revista Cubana de Ingeniería, 8(1), 24-29.

C2020 por los autores. Este artículo es de acceso abierto y distribuido según los términos y condiciones de la licencia Creative Commons Atribución-NoComercial-Compartirlgual 4.0 Internacional (CC BY-NC-SA 4.0)

(https://creativecommons.org/licenses/by-nc-sa/4.0/). 